\title{
Alveolar Epithelial Cells in Mycobacterium tuberculosis Infection: Active Players or Innocent Bystanders?
}

\author{
Julia M. Scordo ${ }^{a}$ Daren L. Knoell ${ }^{b, c}$ Jordi B. Torrelles ${ }^{a, c}$ \\ a Department of Microbial Infection and Immunity, College of Medicine, ${ }^{b}$ Department of Pharmacy, College of \\ Pharmacy, and ${ }^{C}$ Center for Microbial Interface Biology, The Ohio State University, Columbus, Ohio, USA
}

\section{Key Words}

Alveolar epithelial cell · Tuberculosis · Lung environment · Innate immunity

\begin{abstract}
Tuberculosis (TB) is a disease that kills one person every $18 \mathrm{~s}$. TB remains a global threat due to the emergence of drugresistant Mycobacterium tuberculosis (M.tb) strains and the lack of an efficient vaccine. The ability of M.tb to persist in latency, evade recognition following seroconversion, and establish resistance in vulnerable populations warrants closer examination. Past and current research has primarily focused on examination of the role of alveolar macrophages and dendritic cells during M.tb infection, which are critical in the establishment of the host response during infection. However, emerging evidence indicates that the alveolar epithelium is a harbor for M.tb and critical during progression to active disease. Here we evaluate the relatively unexplored role of the alveolar epithelium as a reservoir and also its capacity to secrete soluble mediators upon M.tb exposure, which influence the extent of infection. We further discuss how the M.tb-alveolar epithelium interaction instigates cellto-cell crosstalk that regulates the immune balance between a proinflammatory and an immunoregulatory state, thereby
\end{abstract}

prohibiting or allowing the establishment of infection. We propose that consideration of alveolar epithelia provides a more comprehensive understanding of the lung environment in vivo in the context of host defense against M.tb.

(c) 2015 S. Karger AG, Basel

\section{Introduction}

Tuberculosis (TB), caused by Mycobacterium tuberculosis (M.tb), is the second leading cause of death due to an infectious disease [1]. A multitude of host factors leads to vast interindividual differences in response to M.tb. This is reflected by a wide spectrum of potential outcomes that include total pathogen clearance and host recovery, pulmonary active TB, extrapulmonary active $\mathrm{TB}$, disseminated active TB, and latency with reactivation [2]. It is estimated that half of individuals exposed to M.tb will never develop infection, as assessed by the lack of conversion to a positive skin test [2]. This raises the possibility of host resistance to infection through mechanisms that involve early cell recognition and pathogen clearance.

The most common route of $M$. $t b$ transmission occurs following the deposition of bacilli into alveolar sacs after inhalation [3]. Early infection is founded on the princi-

\section{KARGER}

(C) 2015 S. Karger AG, Basel

$1662-811 \mathrm{X} / 15 / 0081-0003 \$ 39.50 / 0$

E-Mail karger@karger.com

www.karger.com/jin 
pal that upon lung deposition M.tb is phagocytized by alveolar macrophages (AMs) [4] and dendritic cells (DCs) [3]; however, the human lung is comprised of approximately 40 host cell types [5], and how many of these cell populations may become infected is still unknown. In this regard, recent studies have demonstrated that bacterial recognition and uptake can occur via alveolar epithelial cells (ATs), nonprofessional phagocytic cells that line the alveolar tissue [6]. In order to understand the role of ATs during infection, it is necessary to first define the interaction between ATs and M.tb, as well as the subsequent crosstalk between $M . t b$-infected ATs and other host cells at different stages of the infection. Examination of the role of ATs in M.tb pathogenesis taking into account these many factors will yield a more thorough understanding of the lung environment in vivo.

\section{The Role of the Alveolar Epithelium during M.tb Infection}

\section{M.tb Invasion of ATs}

The role of the alveolar epithelium may differ greatly based on the stage of M.tb infection (fig. 1a-c). Early M.tb invasion of ATs has been shown to be essential for the establishment of infection [2]. In principal, it is believed that following inhalation 1-3 bacilli deposit within alveolar sacs [7]. In this instance, the contact of $M$.tb with ATs is believed to be early and of a low frequency. In contrast, contact with ATs may happen at a higher frequency following bacilli release from dying, infected phagocytic cells $[7,8]$ or following release from granulomas [9] (fig. 1a-c). Recent evidence supports that $M . t b$ can invade and replicate within alveolar epithelial type II cells (ATII) during infection [4, 7-11]. Invasion could be beneficial to $M . t b$ because ATIIs are nonprofessional phagocytes [2] and may provide a protected intracellular environment conducive to bacterial replication [9]. Early invasion and survival could also facilitate modification of the M.tb envelope surface [12] and gene expression [9], further decreasing recognition by phagocytic cells [9] and thereby enhancing virulence [12]. In the latter scenario, virulent phenotypes of M.tb strains have been shown to correlate directly with their capacity to invade ATIIs and induce necrosis $[7,9,13]$.

\section{M.tb Replication within the Alveolar Epithelium}

ATIIs infected at a low inoculum dose harbor significantly higher intracellular bacterial levels after 4 days in culture, resulting in cytotoxicity [14]. Moreover, M.tb replication in ATIIs is 50-fold higher in comparison to the values in human or mouse macrophages in vitro [8], which is further supported by evidence of active M.tb replication in ATII cell lines for up to 7 days in culture [15]. Studies using cell lines have shown M.tb growth spreading throughout the epithelium from infected necrotic cells on to neighboring cells [8]. In vivo experiments have
Fig. 1. Interaction of M.tb with the alveolar epithelia. Projected models of how ATs interact with M.tb bacilli during the course of infection. a $M$.tb initial infection. $\mathbf{b}$ M.tb released from lysed macrophages. c $M$.t $b$ in cavities. a Primary infection deposits approximately 1-3 M.tb bacilli per alveolus, resulting in an initial infection of ATII or ATI cells. Low-burden M.tb contact with ATs may occur once or multiple times before the infection of AMs is established. M.tb infection of ATs results in initial evasion of phagocyte detection. $M$.tb-induced AT necrosis then promotes translocation across the basement membrane into the interstitium. Alternatively, M.tb may initially replicate within AMs and then increase the extracellular burden and contact with ATs following the release from lysed macrophages (b) or, alternatively, at later stages of lung infection when $M . t b$ is prominent throughout extracellular spaces within the lung (c). In this situation, a high $M$.tb burden may overwhelm ATs, thereby promoting cell death and the subsequent dissemination of bacteria beyond the airway. d ATII-induced modulation of the host immune response in the lung during M.tb infection. Comparison of the proinflammatory (left) vs. the immunoregulatory/immunosuppressive (right) role of $M$.t $b$-infected ATIIs and the ensuing crosstalk with host cell populations present in the alveolus and in the circulation. Upon M.tb infection, chang- es in ATII activation are dependent on many factors that influence the delicate balance toward a proinflammation or immunosuppressive response. Key factors that influence the host response include the bacterial burden in the alveolus, the stage of infection (primary or reinfection), the initial alveolar host cell population being infected by M.tb, differences in host susceptibility to infection, the identity and degree of virulence of the M.tb strain infecting the host, and the host local alveolar environment, among other factors. The combination of factors present and the subsequent response by ATIIs modulate the establishment and outcome of the M.tb infection. A continuous balance of the ATII immune response between a proinflammatory and an immunosuppressive state is critical for control of the infection by the host, while minimizing related tissue damage in the lung. AEP = Alveolar epithelium; ATI = alveolar epithelial type I cell; CD4 = CD4 T cell; CD8 = CD8 T cell; EAM = epithelial apical membrane; EBM = epithelial basement membrane; $\mathrm{M}=$ macrophage; $\mathrm{m}=$ monocyte; $\mathrm{S}=$ surfactant membrane; $\mathrm{TCR}=\mathrm{T}$-cell receptor; $\mathrm{MR} 1=\mathrm{MHC}$ like molecule MR1. Black and dark red colored circles represent secreted immune mediators (colors refer to the online version only). Neutrophils are depicted as multinuclear cells.

(For figure see next page.) 
exhibited early M.tb growth within ATIIs [10], and evidence for the ability of M.tb to replicate within ATIIs in vivo is supported by isolation of bacterial DNA from the alveolar epithelium in deceased, $M . t b$-infected individuals [16]. Furthermore, M.tb DNA has been found in lung epithelial tissue not associated with granulomas [16]. Collectively, these findings authenticate ATIIs as a cellular reservoir for M.tb capable of facilitating rapid bacterial growth while potentially escaping recognition by phagocytes in the alveolus.

\section{AT Cell Death Induction during M.tb Infection}

It has been postulated that M.tb invasion and replication within ATIIs could overwhelm ATs, resulting in cell death and bacterial dissemination [9]. In support of this, $M . t b$ infection of ATIIs has been shown to cause necrosis (in comparison to apoptosis as observed in M.tb-infected macrophages) [9]. It is plausible that ATII infection, replication, and subsequent release following ATII cell death [9] could lead to immune evasion, thereby minimizing the capacity of nearby resident phagocytic cells to become

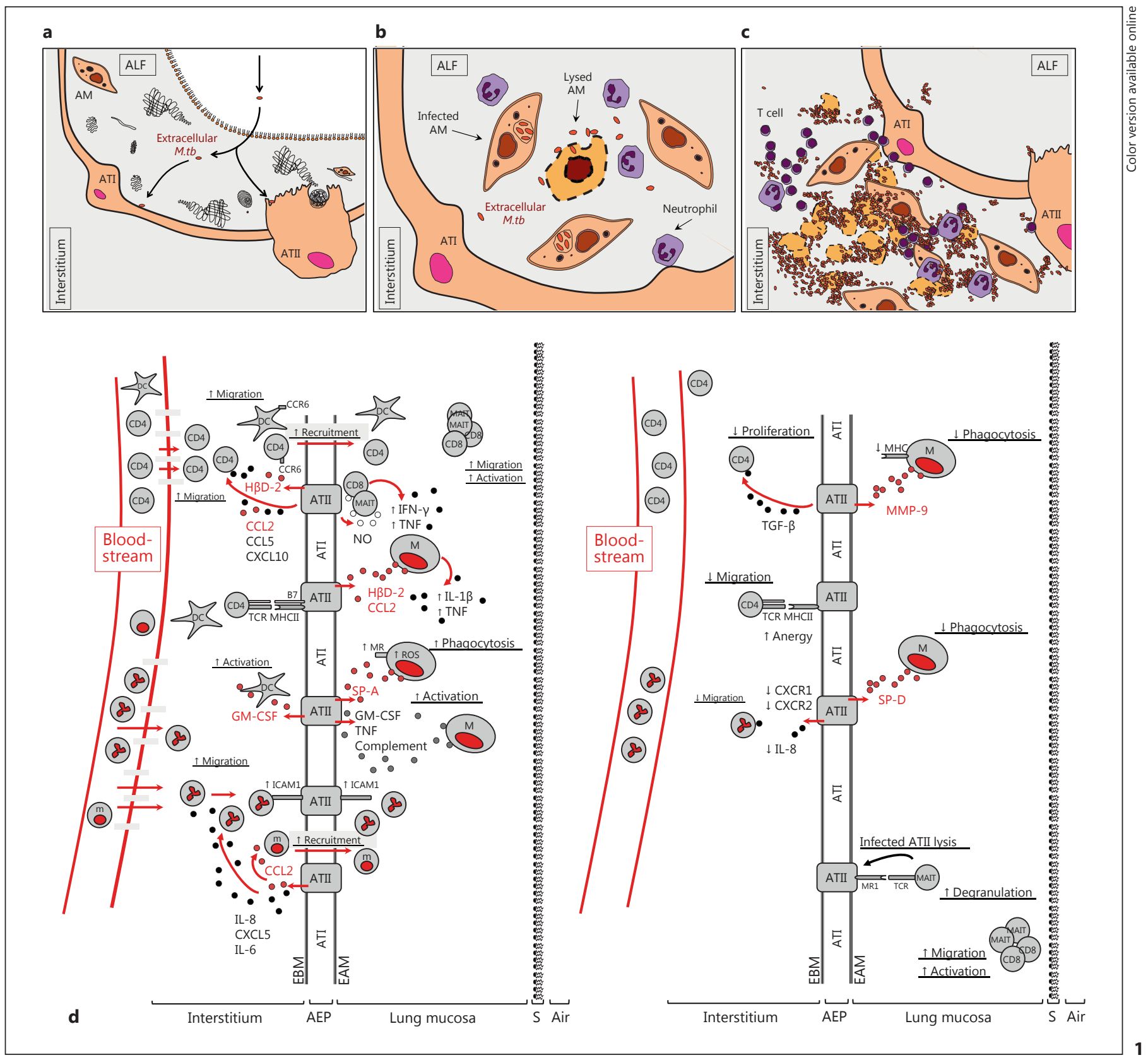

Alveolar Epithelium Function in M.tb 
activated [9]. Presumably, this could allow for enhanced bacterial replication, survival, and dissemination $[4,8,9]$ and potential progression to active disease [9].

While most reports have focused on $M$.tb-induced necrosis in ATs $[2,4,8,9,11,17,18]$, recent work has shown that infection of ATs may also result in apoptosis $[17,19]$. $M . t b$ infection can result in the expression of apoptotic cell markers leading to efferocytosis by resident macrophages [20], which would help to resolve excess inflammation and control further infection. To what extent AT necrosis or apoptosis occurs in vivo remains understudied, but it is likely important because it may contribute to the relatively high interindividual variation in susceptibility to M.tb infection. M.tb replication within ATs with resultant necrosis could substantially increase the burden of $M$. tb bacilli within the alveolar space, leading to infection of neighboring cells, including other ATs or phagocytes [20]. Differences in AT cell death induced by M.tb during infection may also modulate the host immune response and contribute significantly to the propagation or clearance of bacilli.

\section{The Role of ATs in M.tb Dissemination}

While AT uptake of M.tb occurs with a low efficiency, it can result in cell activation and subsequent exocytosis of $M$. $t b$ via the basolateral side of the cell [2]. This suggests the potential for M.tb to permissively enter the apical surface of ATs, cross through the epithelial basement membrane, and migrate into the interstitium before encountering interstitial phagocytic cells. This novel portal of entry counters conventional models of interstitial entry whereby M.tb-infected macrophages [7] and DCs disseminate bacterial infection by travelling from primary granulomas to draining lymph nodes [2]. The guinea pig model of $M$.tb infection supports this concept, showing dissemination of free M.tb to the lymph nodes within days of infection [21]. Clearly the interaction between M.tb and ATs is dynamic, and further examination is important as the M.tb-AT interaction could affect the role of phagocytic cells and alter the infection outcome.

\section{ATs and the Innate Immune Response during M.tb Infection}

\section{AT-Macrophage Interaction}

Primary human ATIIs are capable of producing many relevant biomolecules involved in innate immunity and homeostasis, including complement proteins $\mathrm{C} 2, \mathrm{C} 3, \mathrm{C} 4$, and C5 [20], surfactant lipids, antimicrobial peptides
(AMP) [17], and homeostatic hydrolytic enzymes (termed hydrolases) [22]. ATII secretion of these innate defense biomolecules facilitates cell-to-cell interactions [20], including host cell recruitment, increased inflammation, and/or enhancement of phagocytosis and microbial killing [22-25]. There is substantial evidence for cellular crosstalk between ATs and resident lung macrophages within the alveolar sac [26] (fig. 1d; table 1). This is perhaps best exemplified by the C-type lectin surfactant proteins (SP)-A and SP-D, where SP-A production by ATIIs during M.tb infection has been shown to increase phagocytosis [14] via activation of the phagocytic mannose receptor (MR) on the surface of human macrophages [23]. SP-A also regulates macrophage intracellular killing through enhanced production of reactive oxygen species (ROS) [20] while limiting the nitrogen oxidative response [17]. Many studies support the opsonizing role of SP-A/SP-D in altering macrophage phagocytosis and bacterial clearance $[20,23]$. While in vivo studies using SP-A- and SP-D-deficient mice have revealed no obvious phenotype [17], polymorphisms in human SP-A and SP-D genes have been linked to increased susceptibility to M.tb infection [23]. Interestingly, ATII-secreted biomolecules have been shown to influence polarization of macrophages toward an M2 phenotype (an alternatively activated phenotype) [26], which has been found to more effectively control intracellular growth upon mycobacterial infection [26].

Coculture studies examining direct cell contact between ATIIs and macrophages resulted in decreased M.tb intracellular growth within infected macrophages but no difference in control of $M . t b$ infection in ATIIs [10]. The decreased bacterial replication within infected macrophages was attributed to tumor necrosis factor (TNF) and granulocyte macrophage colony-stimulating factor (GMCSF) secreted by $M$.tb-infected ATIIs [10]. TNF production by macrophages and ATIIs [25] during M.tb infection may also result in harmful effects on the host [25]. In this regard, TNF production can cause ATII necrosis [7] with subsequent $M . t b$ penetration of the alveolar epithelium and endothelium [25]. Furthermore, M.tb penetration of the alveolar epithelium and subsequent transmigration of the endothelium may be enhanced following release from infected macrophages [7]. Accordingly, ATmacrophage interactions may result in drastically different outcomes during M.tb infection, whereby the order in which host cell populations contact $M$.tb and are infected may alter the bacterial virulence and the innate immune response (fig. 1d).

TNF production by $M . t b$-infected macrophages also induces ATII production of the AMP human $\beta$-defensin- 2 
Table 1. AECII crosstalk with host immune cells during $M . t b$ infection

\begin{tabular}{|c|c|c|}
\hline M.t $b$-infected-AECII response & Crosstalk interaction $/ M . t b$ infection outcome & References \\
\hline Production of SP-A & Increased macrophage phagocytosis, activation via MR & 14,23 \\
\hline Production of SP-A & Increased macrophage ROS production & 20 \\
\hline Production of SP-D & Decreased macrophage phagocytosis via bacterial aggregation & 17,23 \\
\hline Production of complement proteins & Increased host cell recruitment; increased phagocytosis & 20 \\
\hline Production of TNF and GM-CSF & Increased macrophage intracellular killing & 10 \\
\hline Necrosis, production of $\mathrm{H} \beta \mathrm{D}-2$ & Macrophage TNF production & 27 \\
\hline Production of CCL-2 & Macrophage IL- $1 \beta$ and TNF production & 17,20 \\
\hline Production of MMP-9 & $\begin{array}{l}\text { Increased macrophage recruitment, decreased macrophage phagocytosis and } \\
\text { antigen-presenting cell capabilities }\end{array}$ & 28 \\
\hline Production of CCL-2 & Increased monocyte migration & 4 \\
\hline Production of IL-8 and CXCL5 & Increased neutrophil recruitment & $13,17,24$ \\
\hline Production of IL-6 & Altered granulocyte differentiation & 20 \\
\hline Increased ICAM-1 surface expression & Increased neutrophil recruitment & 24,30 \\
\hline Necrosis & Neutrophil binding to AECII surface integrins & 20 \\
\hline $\begin{array}{l}\text { During infection and/or activation by } \\
\text { TNF }\end{array}$ & $\begin{array}{l}\text { Translocation across the endothelial layer, increased endothelial surface } \\
\text { molecule expression }\end{array}$ & 25 \\
\hline $\begin{array}{l}\text { Production of CCL-2, CXCL10, and } \\
\text { CCL-5 }\end{array}$ & Increased $\mathrm{CD} 4^{+} \mathrm{T}$ cell recruitment & $13,17,33$ \\
\hline Production of $\mathrm{H} \beta \mathrm{D}-2$ & Increased recruitment of memory T cells and DCs via CCR6 binding & 17 \\
\hline Production of GM-CSF & Activation of DCs in circulation & 20 \\
\hline MHCII surface expression & Increased $\mathrm{CD} 4^{+} \mathrm{T}$ cell activation & 20,34 \\
\hline Costimulatory molecule expression & $\begin{array}{l}\text { Increased T cell activation via antigen-presenting cells, induction of } \mathrm{T} \text { cell } \\
\text { anergy, induction of memory } \mathrm{T} \text { cell populations }\end{array}$ & $\begin{array}{l}6,20,32 \\
34,35\end{array}$ \\
\hline Production of TGF- $\beta$ & Decreased T cell proliferation & 20 \\
\hline $\begin{array}{l}\text { Decreased CXCR } 1 / 2 \text { expression and } \\
\text { IL-8 production }\end{array}$ & Decreased neutrophil recruitment & 31 \\
\hline
\end{tabular}

(H $\beta \mathrm{D}-2)$ [27], which has a prominent role during infection and inflammation. Other TNF-induced AT-secreted factors, that include chemokines CCL-2 and CCL-5, GMCSF, and SP, modulate macrophage growth, migration, and function $[20,25]$. ATII also constitutively express the interferon (IFN)- $\gamma$-inducing factor interleukin (IL)-18 [17], which is elevated in the lung tissue of patients with active TB [17], promoting the $\mathrm{Th}_{1}$ immune response that drives granuloma formation [17]. Another important immune mediator produced by ATII is the enzyme matrix metallopeptidase 9 (MMP-9) [28]. MMP-9 functions to recruit granuloma-forming macrophages during $M$. $t b$ infection; however, the early macrophage population recruited by AT-produced MMP-9 is less capable of phagocytosis and antigen presentation (fig. 1d), thus allowing mycobacterial survival [28]. Other important biomolecules secreted by $M$.tb-infected ATII (i.e. CCL- 2 and IL8) function to generate a chemoattractant gradient on both the basal and the apical regions of the epithelium. Establishment of this gradient plays a vital role in cell recruitment and phagocyte activation in both the alveolus and the bloodstream (fig. 1d). ATs may play an important

Alveolar Epithelium Function in M.tb Infection immunomodulatory role in maintenance of epithelial integrity during $M . t b$ infection by limiting inflammation and inhibiting resident macrophages. This has been observed to occur through TGF- $\beta$ binding to macrophages via epithelial surface integrins [29]. While TGF- $\beta$ production by ATs remains unknown in vivo, this may potentially contribute to the containment of $M . t b$ within granulomas through increased monocyte and macrophage recruitment to the infection site [29]. However, tight regulation is necessary as the antiproliferative effects of excessive TGF- $\beta$ production may induce cytotoxicity, leading to a loss of alveolar structural integrity and promoting mycobacterial growth and dissemination [29].

\section{AT-Neutrophil Interaction}

IL- 8 acts as a chemoattractant by recruiting neutrophils from the circulation to the site of infection [13]. ATIIs produce IL-8 early during M.tb infection $[4,14]$ independently of the presence of proinflammatory stimuli [13]. IL-8 then enhances the response to soluble secreted factors elicited from $M$.tb-infected monocytes, indicative of increased crosstalk between cells within the 
alveolar compartment [14]. IL- 8 is also produced and secreted by resident macrophages and infiltrating neutrophils, and it is typically highly abundant in bronchoalveolar lavage fluid from TB-infected individuals [14]. Recruitment of neutrophils is also driven by CXCL5, a chemokine that is secreted by $M . t b$-infected ATIIs [24]. ATII production of IL- 6 also plays a role in crosstalk with neutrophils during inflammation [20], in which case IL-6 may control granulocyte differentiation and the inflammatory response of granulocytic host cells [20] (fig. 1d).

Intercellular adhesion molecule 1/CD54 (ICAM-1) is an ATII surface adhesin that facilitates crosstalk between ATIIs and neutrophils. Upregulation of ICAM-1 during $M . t b$ infection, under conditions of oxidative stress and in response to proinflammatory cytokines such as TNF [4], has been reported [30]. The induction of ICAM-1 coupled to ATII activation and increased IL-8, CXCL5, and IL- 6 production may account for the substantial number of neutrophils observed in the lungs of active TB patients [24]. In this regard, neutrophil binding to ATII surface integrins can lead to induction of epithelial cell death, thereby potentially contributing to the spread of $M . t b$ infection as previously discussed [20]. It is also plausible that $M . t b$ can evade the early response to infection by inhibiting neutrophil migration to the alveolus through decreased ATII surface expression of CXCR1 and CXCR2 as shown with $M$. bovis BCG [31]. While neutrophil infiltration during $M . t b$ infection can increase the collateral tissue damage in the lung, leading to reactivation or active $\mathrm{TB}$, their presence is also required for innate host defense for early control of bacterial growth. Further studies are required to determine the role that ATs play in regulating neutrophil function in response to M.tb.

\section{The Role of ATs in Initiating Adaptive Immunity}

ATs modulate the function of host cells residing in the alveolus and lung interstitium, suggesting that ATs shape the outcome of infection within and adjacent to the alveolar microenvironment [26]. ATIIs in all likelihood also modulate the presence and activity of other cell populations present in the circulation (i.e. DCs and T cells), thereby providing a bridge between the innate and adaptive immune systems [26, 27]. The alveolar epithelial basement membrane is in direct contact with the lung interstitium, which contains DCs and abundant numbers of lymphocytes [32]. Studies support a role for ATII in initiating the adaptive immune response through CCL-2 (or MCP-1), CXCL-10 (or IP-10), and CCL-5 (or RAN-
TES) [17] production and subsequent recruitment of $\mathrm{CD}^{+} \mathrm{T}$ cells $[13,33]$ (fig. 1d). The recruitment of $\mathrm{T}$ cells is essential for granuloma formation [13]. In this context, $\mathrm{H} \beta \mathrm{D}-2$ produced by ATII can also recruit DCs and memory $\mathrm{T}$ cells by binding to the chemokine receptor CCR6 expressed on the surface of these cells [17]. Furthermore, ATII release of GM-CSF into the basolateral milieu activates circulating DCs and enhances their capacity to activate $\mathrm{T}$ cells in draining lymph nodes [20] (fig. 1d).

ATIIs express major histocompatibility complex class I and II (MHC-I and MHC-II) $[34,35]$ and are capable of initiating adaptive immunity through antigen-mediated contact with T cells $[20,34]$. The expression of MHC-II increases following stimulation with IFN- $\gamma$, which also increases the nitric oxide (NO) production induced by iNOS expression that is sufficient to kill M.tb in the presence of proinflammatory stimuli [25]. Moreover, M.tbinfected ATIIs produce and secrete IFN- $\gamma$ and increase IFN- $\gamma$ receptor expression, thereby creating a positive feedback loop [25] that may assist with initiation of the $\mathrm{Th}_{1}$ response and drive granuloma formation during $M . t b$ infection. In this scenario, $M$. $t b$-induced production of IFN- $\gamma$ from different cells (including macrophages, monocytes, and ATII) may culminate in synergy that minimizes $M$.tb intracellular growth as well as increases antimicrobial activity.

ATIIs also express cell surface costimulatory molecules that coordinate antigen presentation to $\mathrm{CD} 4^{+} \mathrm{T}$ cells $[6,32]$. These factors can be induced by TNF [20] and include ICAM/CD54, costimulatory molecule/signal for T cell activation CD80 (or B7-1), lymphocyte functionassociated antigen 3 (LFA-3 or CD58), and vascular cell adhesion molecule (or CD106) [6]. Published studies have shown differences in the expression levels of MHCI, MHC-II, and costimulatory molecules in primary cells and cell lines and between species [32, 34]. For example, MHC-II is expressed in primary ATIIs under both resting and stimulated conditions but not in the commonly used A549 cell line [32, 35]. Also, B7 expression is not always observed $[32,35]$. The lack of costimulatory molecule expression or changes in expression throughout AT differentiation and maturation could suggest an immunomodulatory role for ATs during M.tb infection (fig. 1d). In this regard, a lack of $\mathrm{B} 7$ expression can promote $\mathrm{CD} 4^{+} \mathrm{T}$ cell anergy through MHC-II binding to naive T cells without proper costimulation [34]. Immune modulation could temper the inflammatory response and prevent T-cellmediated destruction of lung tissue [34]. This has been corroborated by in vivo studies showing that ATII can present antigen to T cells through MHC-II; however, they 
are unable to activate naive or antigen-specific primed $\mathrm{T}$ cells [35]. Differences in surface molecule expression may be imperative to limit inflammation when faced with innocuous antigens while mounting a proper adaptive immune response after encountering authentic pathogens. Additionally, ATII antigen presentation may arise only when the response is critical, such as during M.tb infection. Further immunomodulation could also occur through the production of ATII TGF- $\beta$, an immune mediator that decreases $\mathrm{T}$ cell proliferation [20] and is required for regulatory $\mathrm{T}$ cell function. Moreover, a role for ATII in activation of memory T cells, which do not require costimulation, has also been suggested [35].

Epithelial cells are also capable of immunosuppression by expressing $\mathrm{T}$ cell regulatory ligands, including programmed cell death ligands 1 and 2 (PDL1 and PDL2), $\mathrm{B} 7-\mathrm{H} 3$, and ICOS-L, among others, during viral infections [36]. Expression of these costimulatory and inhibitory receptors on the alveolar epithelial surface is not known during $M$.tb infection in vivo; however, their expression may allow ATIIs to directly regulate $\mathrm{T}$ cell activation and balance the inflammatory environment in the lung during $M . t b$ infection.

Recently it has been suggested that ATIIs may interact with a nonconventional subset of CD8 T cells termed mucosal associated invariant T (MAIT) cells [37]. AT nonpeptide antigen presentation via MR1 could allow for effector function of this innate-like $\mathrm{T}$ cell population prior to full adaptive immune activation. This AT-MAIT interaction could result in MAIT cell lysis of $M$.tb-infected ATs, thereby minimizing infection [37]. This interaction is shown to drive the release of TNF and IFN- $\gamma$, which in turn induces ATII secretion of NO [37] (fig. 1d).

In summary, ATs may provide an important link between the response to $M$.tb and the subsequent establishment of the adaptive immune response; however, unknowns regarding this potential role require further examination.

\section{M.tb Binding and Invasion of the Alveolar Epithelium}

The results of AT binding and internalization of M.tb following recognition of $M$.tb antigens by host cell surface molecules and receptors are shown in table 2. M.tb-AT interactions may occur: (i) during the initial infection following direct deposition in the alveolus, (ii) following exposure to soluble host factors present within the lung mucosa (i.e. SP and hydrolases), and/or (iii) in advanced infection stages following release from necrotic infected host cell populations present in the lung. In the published literature, the majority (if not all) of the studies address the M.tb-AT interaction mimicking early-stage infection. Thus, how M.tb interacts with ATs in advanced stages of the infection (i.e. during reactivation) remains unanswered. Moreover, it is difficult to discern which M.tb cell wall components play a role in the interaction with ATs at specific stages of infection, as there is limited investigation in this area. Accordingly, in this section we present and discuss what is known and what these interactions may mean for the establishment of infection and the ensuing immune response.

During the initial infection following direct deposition in the alveolus, ATIIs internalize M.tb via macropinocytosis $[6,16,38]$ in both a receptor-mediated and an actindependent manner. The process of bacterial uptake is highly coordinated and involves membrane ruffling, microtubule and microfilament polymerization and subsequent signal transduction, bacterial entry, and innate immune activation [6]. Macropinocytosis results in the formation of macropinosomes; however, the killing capacity of these vacuoles is much less efficient than that of phagosomes [16]. Alternative routes of M.tb internalization and trafficking within the alveolar epithelium remain to be elucidated; however, observations suggest that M.tb utilizes different host pathways in ATIIs, in comparison to phagocytes [39]. In this regard, evidence suggests that autophagy pathways are involved within infected ATIIs and promote bacterial survival [12].

ATIIs express a variety of molecules that have been shown to participate in receptor-mediated endocytosis following recognition of M.tb antigens, such as integrins [6], carbohydrate receptors and extracellular matrix (ECM) components [40-43], and pattern recognition receptors [6]. Integrins, including CD51 (vitronectin) and CD29 ( $\beta_{1}$ integrin), have been shown to synergize and be involved in M.tb invasion [6]. The expression of these receptors on the AT surface is highly specific, coordinated, and polarized, which allows for the alveolar epithelium to respond and interact with pathogens and other host cells based on the unique extracellular environment in which it resides.

Surface carbohydrate receptors such as heparin, heparin sulfate, and hyaluronic acid bound to ECM molecules may also allow M.tb access to ATIIs through multiple routes, thereby enhancing their susceptibility to pathogen invasion $[6,43]$. For example, laminin-binding protein (LBP) was first implicated in host cell binding during infection with $M$. leprae and has since been shown to facilitate $M . t b$ binding to the alveolar epithelium through hyaluronic acid, laminin, and collagen [43]. 
Table 2. $M . t b$ antigen interaction and modulation of the AECII immune response

\begin{tabular}{|c|c|c|c|c|c|}
\hline M.tb antigen & Antigen source & Cell type & Target host receptor/molecule & Immune response & Ref. \\
\hline ManLAM & Purified antigen & A549 & Not specified & Expression of $\mathrm{H} \beta \mathrm{D}-2$ & 27 \\
\hline PIMs & $\begin{array}{l}\text { Total PIM fraction from } \\
\text { H37Rv }\end{array}$ & A549 & Not specified & $\begin{array}{l}\text { Cytolysis, ROS production, TGF- } \beta \\
\text { production }\end{array}$ & 19 \\
\hline PIMs & $\begin{array}{l}\text { Total PIM fraction from } \\
\text { H37Rv }\end{array}$ & WI26 & Not specified & $\begin{array}{l}\text { Loss of membrane integrity, cytolysis, } \\
\text { induction of apoptosis, ROS } \\
\text { production, TGF- } \beta \text { production }\end{array}$ & 19 \\
\hline ESAT-6 & Purified recombinant protein & A549 & Not specified & Expression and production of IL-8 & 45 \\
\hline ESAT-6 & Purified recombinant protein & $\mathrm{H} 441$ & Not specified & $\begin{array}{l}\text { Expression and production of IL-8, } \\
\text { necrosis }\end{array}$ & 45 \\
\hline ESAT-6 & $\begin{array}{l}\text { Recombinant and native } \\
\text { protein, cell wall fraction of } \\
\text { H37Rv }\end{array}$ & WI26 & Laminin & Cytolysis & 44 \\
\hline ESAT-6 & $\begin{array}{l}\text { Recombinant and native } \\
\text { protein, cell wall fraction of } \\
\text { H37Rv }\end{array}$ & A549 & Laminin & Cytolysis & 44 \\
\hline HBHA & $\begin{array}{l}\text { Purified protein from } M \\
\text { bovis BCG }\end{array}$ & CHO cells & $\begin{array}{l}\text { Sulfated glycoconjugates } \\
\text { (heparin, dextran sulfate, } \\
\text { fucoidan, and chondroitin } \\
\text { sulfate) }\end{array}$ & $\begin{array}{l}\text { Enhanced cell binding (tested in both } \\
\text { BCG and H37Ra), bacterial } \\
\text { aggregation }\end{array}$ & 2,21 \\
\hline LBP & $\begin{array}{l}\text { Protein isolated from } M \text {. bovis } \\
\text { BCG, recombinant purified } \\
\text { protein }\end{array}$ & A549 & Collagen-I & Enhanced cell binding & 43 \\
\hline LAM, $19 \mathrm{kDa}$ & Purified antigen & $\begin{array}{l}\text { Primary } \\
\text { bronchial } \\
\text { epithelial cells }\end{array}$ & TLR-2 & $\begin{array}{l}\text { Decreased surface expression of } \\
\text { CXCR1/CXCR2 and decreased IL- } 8 \\
\text { production }\end{array}$ & 31 \\
\hline MDP1 & $\begin{array}{l}\text { Native protein isolated from } \\
\text { M. bovis BCG (Tokyo strain) }\end{array}$ & A549 & $\begin{array}{l}\text { Heparin, heparan sulfate, } \\
\text { hyaluronic acid }\end{array}$ & $\begin{array}{l}\text { Cell binding, bacterial evasion (tested } \\
\text { in both BCG and } M . t b \text { strains) }\end{array}$ & 6 \\
\hline Malate synthase & $\begin{array}{l}\text { Recombinant purified } \\
\text { protein, cell wall fraction of } \\
\text { H37Rv }\end{array}$ & A549 & Laminin-1 and fibronectin & Enhanced cell binding & 42 \\
\hline $\begin{array}{l}\text { Fibronectin-binding } \\
\text { protein (FAP) }\end{array}$ & $\begin{array}{l}\text { Recombinant protein from } \\
\text { M. avium strain } 101\end{array}$ & $\begin{array}{l}\text { Fibronectin- } \\
\text { coated wells }\end{array}$ & Fibronectin & $\begin{array}{l}\text { Enhanced binding by } M \text {. avium and } \\
\text { BCG }\end{array}$ & 46 \\
\hline Antigen 85 complex & Purified protein & Caco-2 & Fibronectin, elastin & Enhanced cell binding & 40,41 \\
\hline CFP21 & Recombinant purified protein & WI26 & Not specified & $\begin{array}{l}\text { Apoptosis, ROS production, TGF- } \beta \\
\text { production }\end{array}$ & 19 \\
\hline CFP21 & Recombinant purified protein & A549 & Not specified & Apoptosis, TGF- $\beta$ production & 19 \\
\hline $52-\mathrm{kDa}$ protein & $\begin{array}{l}\text { Clones expressing } \\
\text { recombinant protein from } \\
\text { H37Ra }\end{array}$ & $\mathrm{HeLa}$ & Not specified & $\begin{array}{l}\text { Enhanced bacterial invasion and } \\
\text { survival }\end{array}$ & 6 \\
\hline HBHA & M. bovis BCG and M.tb & A549 & Not specified & $\begin{array}{l}\text { Enhanced cell binding, increased } \\
\text { survival }\end{array}$ & 2,6 \\
\hline$\overline{\mathrm{CFP}}$ & $\begin{array}{l}\text { Proteins from } M . t b \text { strains } \\
\text { H37Rv, HN878, and } \\
\text { CDC1551 }\end{array}$ & A549 & Not specified & Formation of lipid raft aggregates & 39 \\
\hline CFP21 & Recombinant purified protein & WI26 & Not specified & $\begin{array}{l}\text { Apoptosis, ROS production, TGF- } \beta \\
\text { production }\end{array}$ & 19 \\
\hline
\end{tabular}

Early secreted antigenic target protein 6 (ESAT-6) is another M.tb antigen capable of binding to laminins on the epithelial cell surface [21], resulting in cell lysis. Reports of ESAT-6 induction of ATII cell cytotoxicity through necrosis rather than apoptosis [21] may allow for bacterial evasion of the immune response [44].

Interestingly, M.tb surface antigens released by infected macrophages have been shown to be cytotoxic to the lung epithelium $[21,45]$. In particular, the M.tb cell wall lipids and lypoglycans [specifically phosphatidylmyo-inositol mannosides (PIMs) and mannose-capped lipoarabinomannan (ManLAM)] have been shown to result in epithelial cytotoxicity [19]. It is interesting to note that ManLAM induces expression of the AMP $\mathrm{H} \beta \mathrm{D}-2$, showcasing two vastly different immune responses following antigen exposure [27]. Additionally, PIM exposure induces AT cytotoxicity and TGF- $\beta$ production [19]. 
The M.tb antigen heparin-binding hemagglutinin (HBHA) binds to ATIIs $[2,21]$ via sulphated glycoconjugates present on the epithelial cell surface [21]. Binding of this immunogenic antigen allows for $M . t b$ to cross the epithelial barrier without disrupting the monolayer, suggesting that it is taken up through an endocytic pathway [21]. In vivo inhibition of HBHA or mutated HBHA$M . t b$ strains shows fewer M.tb bacilli located outside the lung compartment, supporting a role for this M.tb antigen in dissemination [21]. In contrast, HBHA does not bind to phagocytes, suggesting a unique relationship between this antigen and ATIIs [43].

Another antigen implicated in binding to the ATII ECM, specifically laminin and fibronectin, is the M.tb enzyme malate synthase, which is both expressed on the cell surface and secreted [42]. Antibodies generated against M.tb malate synthase have been observed in individuals with active TB disease [42] and may have therapeutic potential to prevent cellular entry. Interestingly, M.tb malate synthase is unique in that the secreted form is able to reassociate with the M.tb cell wall and promote ATII adherence [42].

The presence of multiple fibronectin-binding proteins on the surface of $M . t b$, including fibronectin attachment protein (FAP) and antigen 85 complex, implicates an important role in bacterial attachment $[40,46]$. Interestingly, the fibronectin binding site targeted by M.tb FAPs is unique in comparison to the regions bound by other pathogens [46].

Invasion inhibition studies conducted using established ATII cell lines, e.g. A549 cells, have identified several other high-affinity binding peptide sequences (HABPs) located in the cell wall of M.tb, and hence exploitation of high-affinity binding peptide sequences to inhibit invasion of $M$. $t b$ has potential as a therapeutic option [6].

The major pattern recognition receptors implicated in M.tb recognition by ATs include Toll-like receptors (TLRs) and C-type lectins, with both receptor families resulting in modulation of the AT immune response during infection. ATs are capable of expressing up to 11 TLRs, with differential distribution on the cell surface due to membrane polarization [6]. Expression levels vary, with TLR- 2 and TLR- 6 being the most abundantly expressed [17]. Relative to M.tb, TLR-2, TLR-4, TLR-6, and TLR-9 have been shown to influence AT-pathogen interactions [17]. AT activation through TLRs is linked to the production of immune mediators, including chemokines, cytokines, and AMP [17]. Expression of TLR-2 and TLR-6 is observed primarily on the alveolar epithelium apical

Alveolar Epithelium Function in M.tb

Infection membrane [17]. ATII recognition of multiple $M . t b$ antigens through TLR-2 activation [i.e. PIMs, ManLAM, lipomannan, and lipoprotein $19 \mathrm{kDa}$ ] leads to increased production of the neutrophil chemoattractant CXCL-5 [24]. Interaction of M.tb with TLR-2 also induces production of AMPs, including H $\mathrm{BD}-2$ and cathelicidin, i.e. LL37 [17]. Increased AMP production has been shown to further increase ATII TLR-2, TLR-4, and TLR-9 expression via autocrine feedback loops [17]. The role of TLR-4 in AT signaling remains less clear but has been shown to be primarily located within intracellular stores [29].

The C-type lectin Dectin-1 is well known for its affinity to $\beta$-glucans [23]; however, recent studies have shown that Dectin-1 is capable of triggering immune activation, bacterial uptake, intracellular killing, and production of ROS $[6,17,23]$, chemokines, and cytokines [17, 23]. Dectin-1 binds to a yet-to-be-identified M.tb antigen(s) that triggers ATIIs ROS production [17]. Cellular activation through this pathway also increases IL-12 and AMP production, which decreases bacterial intracellular growth in ATIIs [17]. M.tb infection of ATIIs also induces a Dectin1 -dependent increase in IL-8, TNF, and IL-6 expression [6]. Dectin-1 has been suggested to work in conjunction with TLR-2 [23] in M.tb recognition and induction of the innate immune response by ATIIs [17]. Accordingly, M.tb-induced inhibition of Dectin-1 expression in ATIIs may have the capacity to downregulate the proinflammatory response, resulting in poor recruitment of the host cell populations necessary for antimicrobial activity and thereby delaying granuloma formation.

Following cellular entry, virulent $M$.tb drives phagosome maturation arrest in phagocytes [12, 47]. In sharp contrast, M.tb uptake and compartmentalization into ATII vesicles reach full maturity and phenotypically resemble late rather than early endosomes. Although M.tb does not block phagosome maturation in ATIIs, M.tbcontaining late endosomes within ATIIs fail to acidify, thereby allowing $M . t b$ to evade destruction and replicate $[12,19]$. Autophagy has also been suggested as an outcome following M.tb internalization by ATs; however, autophagy has been shown to promote $M$.tb growth within infected ATIIs suggesting that M.tb may have evolved to utilize AT cellular machinery to avoid elimination [12].

The interaction between ATs and the M.tb cell wall surface or released cell wall components may also occur through less direct means. For example, ATII-produced SP-A/SP-D are capable of binding ManLAM [23]. We have shown that ManLAM is released from the M.tb cell surface into the milieu (up to $70 \%$ release) by the action of host alveolar hydrolases present in human lung mu- 
cosa [22]. ManLAM molecules released from the M.tb cell surface may hijack soluble SP-A/SP-D or unmask novel motifs on the M.tb cell wall surface that favor SP-A/SP-D binding to M.tb. Lung mucosa hydrolases together with other innate components, such as SP-A/SP-D, could thereby directly or indirectly orchestrate M.tb phagocytosis by host cells [22]. In this regard, extensive studies of the role of SP-A/SP-D through interaction with M.tb exhibit a high degree of interindividual differences in the host immune response [23]. This suggests that the establishment and progression of infection may depend on the content, timing, and extent to which AT-secreted innate mediators interact with M.tb bacilli in the lung.

\section{ATs Research Limitations}

This review provides a comprehensive assessment of the interaction between alveolar epithelia and M.tb. While substantial evidence of this interaction has increased our understanding of the role of ATs during $M . t b$ infection, contradictory findings underscore the importance of continued study within this field. Findings were obtained through a variety of models that include primary human and animal cells, animal models, and cell lines. Research conducted with primary human ATs would best represent the lung epithelium; however, the main limitation in such research is the capacity to study intact lung tissue in humans. Also, primary ATIIs serve as progenitors for ATIs resulting in a mixed phenotype with prolonged culture [48], although recently it was shown that the ATII phenotype can be maintained in primary cell cultures through serum condition manipulation [48]. Difficulties obtaining and utilizing primary cells have led investigators to commercially available cell models with potential advantages that include removal of donor variability, increased culture life, and a lower cost; however, these models also have limitations due to altered phenotypes that do not fully resemble primary ATII cells. Use of transformed cell lines, such as the commonly used human A549 type II-like cell line, harbors differences in gene and protein expression and response to external stimuli when compared to primary human AT [48]. For example, the role of ATIIs in initiating adaptive immunity remains to be substantiated in part due to differences in the expression of MHCII and costimulatory molecules observed between primary cells, cell lines, and animal models [32,35]. Animal models do not fully replicate the complexity of the human epithelium due to differences in the immune response to pathogens, as well as anatomical and physiological differences. This is perhaps best exemplified in granuloma formation [3], and the contribution of ATs to the establishment of granuloma formation and maintenance remains unknown.

Finally, in an effort to expand our understanding of the role of ATs within the lung microenvironment, coculture studies have revealed the capacity of ATIIs to communicate with other resident and/or recruited cells. These studies have clearly revealed that ATIIs alter the alveolar landscape either following M.tb invasion or subsequent to pathogen entry into other cells. However, the extent of crosstalk between ATIIs and other immune cells and how this shapes the course of $M$. $t b$ infection requires further investigation.

\section{Concluding Remarks}

Herein, we have provided a comprehensive assessment of the roles of ATs as nonphagocytic cells with the potential to influence host defense and pathogenesis following M.tb inhalation into the lung. We postulate that ATs play a role in both the direct recognition and internalization of $M$.t $b$ bacilli and in modulating the immune response of host cell populations within the lung.

Whether infection of the alveolar epithelium is restricted to early inoculation or, more likely, occurs throughout the spectrum of the disease is unknown. With regard to the latter, invasion of ATs may occur following release from dying, infected phagocytes. Accelerated M.tb intracellular growth and the release of AT inflammatory mediators may result in enhanced host susceptibility. It is plausible that the incapacity of ATIIs to efficiently kill $M . t b$ once inside the cell may promote further growth consequent to AT necrosis, allowing M.tb to infect neighboring cells. Alternatively, based on our unpublished results where M.tb exposed to human lung mucosa is better controlled by ATIIs, these cells may potentially act as a refuge for M.tb during establishment of the infection, facilitating a slow but steady replication, evasion of detection by phagocytes, and minimization of an aggressive inflammatory response and tissue damage. This may allow M.tb to adapt by limiting its metabolism and growth prior to reactivation of a latent infection and progression to active disease. Restructuring of the M.tb cell wall is plausible, as we recently showed that ATII lamellar bodies contain an array of hydrolases that significantly modify the M.tb cell wall $[22,49]$. The physiological adaptations of $M$.tb to the contents of these lamellar bodies and the
12

Innate Immun 2016;8:3-14 DOI: $10.1159 / 000439275$
Scordo/Knoell/Torrelles 
ensuing biological consequences during infection are unknown and are currently under further investigation. Lastly, ATs could promote dissemination of infection by providing $M . t b$ access to the interstitial space via apical entry and basolateral exocytosis. In this regard, M.tb may interact with interstitial DCs, which then migrate to the lymph nodes to prime the adaptive immune response.

In conclusion, a more comprehensive examination of the role of ATIIs relative to their capacity to directly interact with M.tb, to communicate with other cells within the alveolar compartment, and to impact the host immune response is required to more thoroughly understand the outcome of M.tb infection. This is important because further elucidation of AT-M.tb interactions has the potential to reveal novel 'druggable' pathways as well as further enhance our capacity to predict the host susceptibility and host response once infection has occurred.

\section{Acknowledgements}

The writing of this review was supported by The Ohio State University College of Medicine Systems and Integrative Biology (SIB) Training Program Fellowship from the National Institutes of Health (NIH)/National Institute of General Medical Sciences (NIGMS) (T32-GM068412 to J.M.S.); the NIH/National Heart, Lung and Blood Institute (NHLBI) (HL118268 to D.L.K.), and the NIH/National Institute of Allergy and Infectious Diseases (NIAID) (AI073856 and AI093570 to J.B.T.).

\section{References}

1 World Health Organization: WHO tuberculosis fact sheet 2015. http://www.who.int/ mediacentre/factsheets/fs104/en/ (accessed March 20, 2015).

-2 Russell DG: TB comes to a sticky beginning. Nat Med 2001;7:894-895.

-3 Sasindran J, Torrelles JB: Mycobacterium tuberculosis infection and inflammation: what is beneficial for the host and for the bacterium? Front Microbiol 2011;2:1-16.

$\checkmark 4$ Bermudez LE, Sangari FJ, Kolonoski P, Petrofsky M, Goodman J: The efficiency of the translocation of Mycobacterium tuberculosis across a bilayer of epithelial and endothelial cells as a model of the alveolar wall is a consequence of transport within mononuclear phagocytes and invasion of alveolar epithelial cells. Infect Immun 2002;70:140-146.

5 van Golde LM: Synthesis of surfactant lipids in the adult lung. Annu Rev Physiol 1985;47: 765-774.

6 Garcia-Perez BE, Castrejon-Jimenez NS, Luna-Herrera J: The role of non-phagocytic cells in mycobacterial infections; in Cardona PJ (ed): Understanding Tuberculosis - Analyzing the Origin of Mycobacterium tuberculosis Pathogenicity. Rijeka, InTech, 2014, pp 149178.

7 McDonough KA, Kress Y: Cytotoxicity for lung epithelial cells is a virulence-associated phenotype of Mycobacterium tuberculosis. Infect Immun 1995;63:4802-4811.

-8 Castro-Garza J, King CH, Swords WE, Quinn FD: Demonstration of spread by Mycobacterium tuberculosis bacilli in A549 epithelial cell monolayers. FEMS Microbiol Lett 2002;212: 145-149.

$\checkmark 9$ Dobos KM, Spotts EA, Quinn FD, King CH: Necrosis of lung epithelial cells during infection with Mycobacterium tuberculosis is preceded by cell permeation. Infect Immun 2000; 68:6300-6310.

Alveolar Epithelium Function in M.tb Infection
10 Sato K, Tomioka H, Shimizu T, Gonda T, Ota F, Sano C: Type II alveolar cells play roles in macrophage-mediated host innate resistance to pulmonary mycobacterial infections by producing proinflammatory cytokines. J Infect Dis 2002;185:1139-1147.

11 Castro-Garza J, Swords WE, Karls RK, Quinn FD: Dual mechanism for Mycobacterium tuberculosis cytotoxicity on lung epithelial cells. Can J Microbiol 2012;58:909-916.

12 Fine KL, Metcalfe MG, White E, Virji M, Karls RK, Quinn FD: Involvement of the autophagy pathway in trafficking of Mycobacterium tuberculosis bacilli through cultured human type II epithelial cells. Cell Microbiol 2012;14:1402-1414.

13 Lin Y, Zhang M, Barnes PF: Chemokine production by a human alveolar epithelial cell line in response to Mycobacterium tuberculosis. Infect Immun 1998;66:1121-1126.

14 Wickremasinghe MI, Thomas LH, Friendland JS: Pulmonary epithelial cells are a source of IL-8 in the response to Mycobacterium tuberculosis: essential role of IL-1 from infected monocytes in a NF- $\kappa \mathrm{B}$-dependent network. J Immunol 1999;163:3936-3947.

15 Bruns H, Stenger S: New insights into the interaction of Mycobacterium tuberculosis and human macrophages. Future Microbiol 2014; 9:327-341.

16 Garcia-Perez BE, Mondragon-Flores R, Luna-Herrera J: Internalization of Mycobacterium tuberculosis by macropinocytosis in nonphagocytic cells. Microb Pathog 2003;35:4955.

17 Li Y, Wang Y, Liu X: The role of airway epithelial cells in response to mycobacteria infection. Clin Dev Immunol 2012;2012:791392.

18 Birkness KA, Deslauriers M, Bartlett JH, White $\mathrm{EH}$, King $\mathrm{CH}$, Quinn FD: An in vitro tissue culture bilayer model to examine early events in Mycobacterium tuberculosis infection. Infect Immun 1999;67:653-658.
19 Vir P, Gupta D, Agarwal R, Verma I: Immunomodulation of alveolar epithelial cells by Mycobacterium tuberculosis phosphatidylinositol mannosides results in apoptosis. APMIS 2014;122:268-282.

20 Fehrenbach H: Alveolar epithelial type II cell: defender of the alveolus revisited. Respir Res 2001;2:33-46.

-21 Krishnan N, Robertson BD, Thwaites G: The mechanisms and consequences of the extrapulmonary dissemination of Mycobacterium tuberculosis. Tuberculosis (Edinb) 2010;90: 361-366.

-22 Arcos J, Sasindran SJ, Fujiwara N, Turner J, Schlesinger LS, Torrelles JB: Human lung hydrolases delineate Mycobacterium tuberculosis-macrophage interactions and the capacity to control infection. J Immunol 2011;187: 372-381

23 Torrelles JB, Azad AK, Henning LN, Carlson TK, Schlesinger LS: Role of C-type lectins in mycobacterial infections. Curr Drug Targets 2008;9:102-112.

24 Nouailles G, Dorhoi A, Koch M, et al: CXCL5secreting pulmonary epithelial cells drive destructive neutrophilic inflammation in tuberculosis. J Clin Invest 2014;124:1268-1282.

$25 \mathrm{Wu}$ L, Guangcun D, Min L, Xiaoming L, Wang Y: Roles of mucosal immunity against Mycobacterium tuberculosis infection. Tuberc Res Treat 2012;2012:1-12.

26 Chuquimia OD, Petursdottir DH, Periolo N, Fernandez C: Alveolar epithelial cells are critical in protection of the respiratory tract by secretion of factors able to modulate the activity of pulmonary macrophages and directly control bacterial growth. Infect Immun 2013; 81:381-389.

27 Rivas-Santiago B, Schwander SK, Sarabia C, et al: Human $\beta$-defensin 2 is expressed and associated with Mycobacterium tuberculosis during infection of human alveolar epithelial cells. Infect Immun 2005;73:4505-4511.

Infection 
28 Bozzano F, Marras F, De MA: Immunology of tuberculosis. Mediterr J Hematol Infect Dis 2014;6:e2014027.

29 Mayer AK, Dalpke AH: Regulation of local immunity by airway epithelial cells. Arch Immunol Ther Exp (Warsz) 2007;55:353-362.

- 30 Lakshminarayanan V, Beno DW, Costa RH, Roebuck KA: Differential regulation of interleukin-8 and intercellular adhesion molecule- 1 by $\mathrm{H}_{2} \mathrm{O}_{2}$ and tumor necrosis factor- $\mathrm{a}$ in endothelial and epithelial cells. J Biol Chem 1997;272:32910-32918.

-31 Hakansson G, Lutay N, Andersson M, et al: Epithelial G protein-coupled receptor kinases regulate the initial inflammatory response during mycobacterial infection. Immunobiology 2013;218:984-994.

-32 Corbiere V, Dirix V, Norrenberg S, Cappello M, Remmelink M, Mascart F: Phenotypic characteristics of human type II alveolar epithelial cells suitable for antigen presentation to T lymphocytes. Respir Res 2011;12:15.

33 Eghtesad M, Jackson HE, Cunningham AC: Primary human alveolar epithelial cells can elicit the transendothelial migration of CD14+ monocytes and CD3+ lymphocytes. Immunology 2001;102:157-164.

-34 Cunningham AC, Zhang JG, Moy JV, Ali S, Kirby JA: A comparison of the antigen-presenting capabilities of class II MHC-expressing human lung epithelial and endothelial cells. Immunology 1997;91:458-463.

- 35 Lo B, Hansen S, Evans K, Heath JK, Wright JR: Alveolar epithelial type II cells induce T cell tolerance to specific antigen. J Immunol 2008; 180:881-888.
36 Stanciu LA, Bellettato CM, Laza-Stanca V, Coyle AJ, Papi A, Johnston SL: Expression of programmed death-1 ligand (PD-L) 1, PD$\mathrm{L} 2, \mathrm{~B} 7-\mathrm{H} 3$, and inducible costimulator ligand on human respiratory tract epithelial cells and regulation by respiratory syncytial virus and type 1 and 2 cytokines. J Infect Dis 2006;193: 404-412.

37 Gold MC, Napier RJ, Lewinsohn DM: MR1restricted mucosal associated invariant $\mathrm{T}$ (MAIT) cells in the immune response to $M y$ cobacterium tuberculosis. Immunol Rev 2015; 264:154-166.

38 Bermudez LE, Goodman J: Mycobacterium tuberculosis invades and replicates within type II alveolar cells. Infect Immun 1996;64: 1400-1406.

39 Fine-Coulson K, Reaves BJ, Karls RK, Quinn FD: The role of lipid raft aggregation in the infection of type II pneumocytes by Mycobacterium tuberculosis. PLoS One 2012;7:e45028.

40 Kuo CJ, Bell H, Hsieh CL, Ptak CP, Chang YF: Novel mycobacteria antigen 85 complex binding motif on fibronectin. J Biol Chem 2012;287:1892-1902.

41 Kuo CJ, Ptak CP, Hsieh CL, Akey BL, Chang YF: Elastin, a novel extracellular matrix protein adhering to mycobacterial antigen 85 complex. J Biol Chem 2013;288:3886-3896.

42 Kinhikar AG, Vargas D, Li H, et al: Mycobacterium tuberculosis malate synthase is a laminin-binding adhesin. Mol Microbiol 2006; 60:999-1013.
43 Dias AA, Raze D, de Lima CS, et al: Mycobacterial laminin-binding histone-like protein mediates collagen-dependent cytoadherence. Mem Inst Oswaldo Cruz 2012;107(suppl 1):174-182.

44 Kinhikar AG, Verma I, Chandra D, et al: Potential role for ESAT6 in dissemination of $M$. tuberculosis via human lung epithelial cells. Mol Microbiol 2010;75:92-106.

45 Boggaram V, Gottipati KR, Wang X, Samten B: Early secreted antigenic target of $6 \mathrm{kDa}$ (ESAT-6) protein of Mycobacterium tuberculosis induces interleukin-8 (IL-8) expression in lung epithelial cells via protein kinase signaling and reactive oxygen species. J Biol Chem 2013;288:25500-25511.

46 Zhao W, Schorey JS, Groger R, Allen PM, Brown EJ, Ratliff TL: Characterization of the fibronectin binding motif for a unique mycobacterial fibronectin attachment protein, FAP. J Biol Chem 1999;274:4521-4526.

47 Torrelles JB: Broadening our view aobut the role of Mycobacterium tuberculosis cell envelope components during infection: a battle for survival; in Cardona PJ (ed): Understanding Tuberculosis - Analyzing the Origin of $\mathrm{Myco}$ bacterium tuberculosis Pathogenicity. Rijeka, InTech, 2012, pp 1-46

48 Mao P, Wu S, Li J, et al: Human alveolar epithelial type II cells in primary culture. Physiol Rep 2015;3.

49 Arcos J, Diangelo L, Scordo J, et al: Lung mucosa lining fluid modifies Mycobacterium tuberculosis to reprogram human neutrophil killing mechanisms. J Infect Dis 2015, DOI: 110.1093/infdis/jiv146 